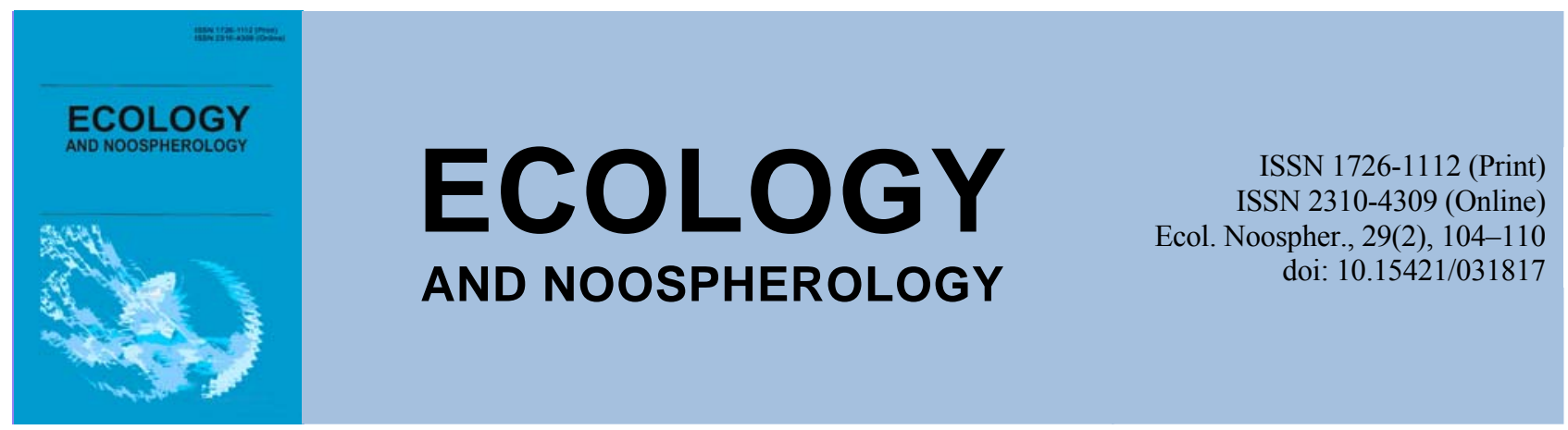

\title{
The method of determining the plants phytoindicator traits adaptive changes coefficient
}

\author{
E. G. Tyulkova, L. P. Avdashkova \\ Belarusian trade and economic university of consumer cooperatives, Gomel, Belarus
}

Article info

Received 03.09.2018

Received in revised form

12.09.2018

Accepted 15.09.2018

Belarusian trade and economic university of consumer cooperatives,

October ave., 50, Gomel, 246029, Belarus.

Tel.: +375-29-316-44-83

E-mail:tut-3@mail.ru
Tyulkova, E. G., \& Avdashkova, L. P. (2018). The method of determining the plants phytoindicator traits adaptive changes coefficient. Ecology and Noospherology, 29(2), $104-110$. doi:10.15421/031817

One of the key environmental problem is the study of protective and adaptive reactions of plants to technogenic conditions. When carrying out a comparative analysis of plants implementation results adaptive mechanisms the quantitative assessment of these processes is great importance. Currently, calculation formulas have been developed to assess the adaptive potential of a particular cultivar, taking into account their yield. In this case, it is necessary to use the average yield of the variety and culture for the entire period of cultivation to quantify the value of the adaptive potential realization. There is a graphical method of determining the value of adaptive potential plants implementation using curves variability phytoindicator features of the studied plants (length, width, area, shape index sheet, the annual growth of trees, the chlorophyll fluorescence induction index etc.), growing under man-made conditions, and the curves of plants these signs variability in background conditions. The value of the adaptive capacity implementation in this case may be defined as the percentage area overlap of the variability phytoindicator characteristic the object in technogenic conditions curve and variability phytoindicator the control test object curve. However, this method provides for the contribution of only the adaptive changes degree and does not take into account the depth of differences in the phytoindicator characteristics the object in technogenic conditions and the control object. To assess changes in the plants parameters under the influence adverse factors environment possible with use the analysis method of growth in ontogenesis lamina dynamics in the contaminated territories. The method involves study the parameters average dynamics (length, width, perimeter and leaf area) of leaves groups (at least five in the group), located on hanging branches on the world four sides, depending on the distance from the road edge and the each group leaves height above the soil surface. Further, statistical modeling reveals multifactorial regularities of the influence of the accounting sheet height above the soil surface, the distance from the road to the accounting leaves individual parameters change. Given the adaptive changes genetic nature developed discrete-systematic approach to the plants adaptive capacity analysis, which allows us to analyze data about the adaptive reactions genetic nature and to obtain new information about the management at different levels of living organisms organization, including the biosphere. Since these methods do not provide for the definition a single quantitative criterion that allows comparative analysis of the several plants phytoindicator parameters dynamics, this was the purpose of this work.

Keywords: vegetation; technogenesis; adaptive changes; phytoindicator signs

\section{Способ определения величины коэффициента адаптивных изменений фитоиндикаторных признаков растений}

\author{
Е. Г. Тюлькова, Л. П. Авдашкова
}

Белорусский торгово-экономический университет потребительской коопераџии, Гомель, Беларусь

В статье рассматривается способ количественного определения величины коэффициента адаптивных изменений параметров растений под влиянием факторов внешней среды, в т.ч. техногенных, относительно фоновых условий. Итоговый коэффициент адаптивных изменений предлагается определять как сумму коэффициентов адаптивных изменений 
относительно всех исследуемых параметров, количество которых связано с целями, задачами, техническими возможностями конкретного исследования. Такой подход позволяет рассчитать единый количественный критерий, позволяющий провести сравнительный анализ нескольких исследуемых фитоиндикаторных параметров анализируемых растений.

Ключевые слова: растительность; техногенез; адаптивные изменения; фитоиндикаторные признаки

\section{Введение}

Одной из ключевых экологических проблем на современном этапе является изучение защитноадаптивных реакций растений на техногенные условия. При проведении сравнительного анализа результатов реализации адаптивных механизмов растений большое значение имеет количественная оценка этих процессов.

В настоящее время разработаны расчетные формулы для оценки адаптивного потенциала отдельного сорта культурных растений с учетом их урожайности (Aseeva, 2015). В данном случае для количественной оценки величины реализации адаптивного потенциала необходимо использовать среднюю урожайность сорта и культуры за весь период выращивания.

Существует графический способ определения величины реализации адаптивного потенциала растений с использованием кривых вариабельности фитоиндикаторных признаков исследуемых растений (длина, ширина, площадь, индекс формы листа, годовой прирост деревьев, индекс индукции флуоресценции хлорофилла и др.), произрастающих в техногенных условиях, и кривых вариабельности этих же признаков растений в фоновых условиях (Besel, 2001). Величину реализации адаптивного потенциала в таком случае возможно определить как процент площади перекрывания кривой вариабельности фитоиндикаторного признака исследуемого объекта в техногенных условиях и кривой вариабельности фитоиндикаторного признака контрольного тест-объекта. Однако такой способ предусматривает вклад только степени адаптивных изменений и не учитывает глубину различий фитоиндикаторных признаков объекта в техногенных условиях и контрольного объекта.

Для проведения оценки изменений параметров растений под влиянием неблагоприятных факторов среды возможно использование способа анализа динамики роста в онтогенезе листовых пластинок на загрязненных территориях (Mazurkin, 2016). Способ предусматривает изучение средней динамики параметров (длина, ширина, периметр и площадь листа) групп листьев (не менее пяти в группе), расположенных на висячих ветвях по четырем сторонам света, в зависимости от расстояния от края автомобильной дороги и высоты расположения каждой группы листьев над поверхностью почвы. Далее статистическим моделированием выявляют многофакторные закономерности влияния высоты расположения учетного листа над поверхностью почвы, расстояния от дороги на изменение отдельных исследуемых параметров учетных листьев.

C учетом генетической природы адаптивных изменений разработан дискретно-системный подход к анализу адаптивного потенциала растений, который позволяет анализировать данные о генетической природе адаптивных реакций и получать новую информацию об управлении ими на различных уровнях организации живых организмов, включая биосферный (Zhuchenko, 2008).

Поскольку перечисленные способы не предусматривают определения единого количественного критерия, позволяющего провести сравнительный анализ динамики фитоиндикаторных параметров нескольких растений, это явилось целью данной работы.

\section{Объекты и методы}

Поставленная задача решается путем определения величины коэффициента адаптивных изменений фитоиндикаторных признаков растений на основании наличия площади перекрывания кривых вариабельности признаков в техногенных и фоновых условиях.

Способ включает расчет величины коэффициента адаптивных изменений на основании определения вероятности и коэффициента глубины адаптивных изменений. При этом величина вероятности адаптивных изменений равна площади фигуры, заключенной между графиками плотностей распределения техногенной и фоновой вероятностей, а для определения коэффициента глубины адаптивных изменений необходимо сравнить интервалы наиболее вероятных значений показателя в техногенной зоне и в фоновых условиях. Итоговую величину коэффициента адаптивных изменений устанавливают по следующей формуле:

$$
A \Pi=\sum_{i=1}^{n} P_{i} \times \Gamma_{i},
$$

где $n$ - количество показателей; $P_{i}-$ вероятность адаптивных изменений $i$-го параметра; $\Gamma_{i}-$ коэффициент глубины адаптивных изменений $i$-го параметра.

Таким образом, предлагаемый способ позволяет количественно определить величину коэффициента адаптивных изменений параметров растений под влиянием факторов внешней среды, в т.ч. техногенных, относительно фоновых условий.

\section{Результаты и их обсуждение}

Для проведения количественной оценки величины коэффициента адаптивных изменений на начальном этапе необходимо выявить наличие для каждого исследуемого параметра (например, длина, ширина, площадь, флуктуирующая асимметрия листовой пластинки, зольность и т.д.) различий между значениями в техногенных и фоновых условиях. Для этого вначале проверяется гипотеза о нормальном законе распределения параметров, затем - гипотеза о равенстве дисперсий нормальных выборок (в Excel - двухвыборочный F-тест для дисперсий), далее в зависимости от наличия различий между дисперсиями - гипотезы о равенстве математических ожиданий при неизвестных и равных дисперсиях и о равенстве математических ожиданий при неизвестных и неравных дисперсиях (в Excel двухвыборочный t-тест с одинаковыми или различными дисперсиями).

На предварительном этапе обработки данных по параметрам асимметрии листовых пластинок необходимо убедиться во флуктуирующем характере асимметрии каждого признака и отсутствии или наличии направленной асимметрии и антисимметрии, а также достоверности различий между параметрами листа в техногенной и фоновой зонах и характере корреляционной связи между конкретными параметрами асимметрии листа отдельно с каждой стороны.

Для установления наличия или отсутствия направленной асимметрии проверяется гипотеза о равенстве показателя симметрии на левой и правой стороне листовых пластинок с помощью критерия Колмогорова-Смирнова. Для наличия или отсутствия антисимметрии определяется эксцесс коэффициента флуктуирующей асимметрии, и он проверяется на значимость. Для территорий, значения эксцесса которых положительны, антисимметрия отсутствует. В случае отрицательного эксцесса проверяется гипотеза о его значимости для подтверждения отсутствия антисимметрии. 
Значение предлагаемого коэффициента адаптивных изменений, характеризующих адаптивный потенциал растений, определяется как произведение вероятности и коэффициента глубины адаптивных изменений всех исследованных параметров листа. При этом вероятность характеризует частоту отклонений исследуемых параметров от фоновых значений, т.е. количество фактически адаптированного материала; глубина - во сколько раз значительны эти отклонения по сравнению с фоновыми условиями.

Фигура, заключенная между графиками плотностей распределения вероятностей техногенных и фоновых условий, содержит те значения показателя листовых пластинок техногенной зоны, которые отличаются от фоновых значений и являются адаптированными к данным техногенным условиям. Площадь такой фигуры $P_{i}$ равна вероятности адаптивных изменений листовых пластинок относительно $i$-го параметра.

Значения вероятности адаптивных изменений $(P)$ по каждому параметру находятся как разность между площадью под кривой плотности нормального распределения признака в техногенных условиях, равной 1 , и площадью перекрывания техногенной и фоновой кривых функций плотностей распределения вероятностей $f(x)=\frac{1}{\sigma \sqrt{2 \pi}} e^{-\frac{(x-a)^{2}}{2 \cdot \sigma^{2}}}, \quad$ где $\quad$ а - среднее значение параметров; $\sigma$ - стандартное отклонение параметров в техногенных и фоновых условиях.

Пусть для определенности рассуждений $f_{l}(x)$ плотность распределения, $a_{l}$ - математическое ожидание, $\sigma_{1}$ - стандартное отклонение, $\mathrm{F}_{1}(x)=\int_{-\infty}^{x} f_{1}(x) d x$ интегральная функция распределения параметра фоновых условий, $f_{2}(x)$ - плотность распределения, $a_{2}$ математическое ожидание, $\sigma_{2}$ - стандартное отклонение, $\mathrm{F}_{2}(x)=\int_{-\infty}^{x} f_{2}(x) d x-$ интегральная функция распределения параметра техногенной зоны. Интегральная функция нормального распределения связана с функцией Лапласа $\Phi(x)=\frac{1}{\sqrt{2 \pi}} \int_{0}^{x} e^{-\frac{t^{2}}{2}} d t$, значения которой находятся в MS Excel с помощью функции НОРМ.РАСП. Для вычисления вероятности того, что нормально распределенная случайная величина $\mathrm{X}$ будет принимать значения в промежутке $(\alpha \quad \beta)$, используется формула $P(\alpha \leq X \leq \beta)=\Phi\left(\frac{\beta-a}{\sigma}\right)-\Phi\left(\frac{\alpha-a}{\sigma}\right), \quad$ где $\quad a \quad-$ математическое ожидание; $\sigma$ - среднее квадратическое отклонение.

Для нахождения в общем случае точек пересечения графиков функций $f_{1}(x)=\frac{1}{\sigma_{1} \sqrt{2 \pi}} e^{-\frac{\left(x-a_{1}\right)^{2}}{2 \cdot \sigma_{1}{ }^{2}}} \quad$ и $f_{2}(x)=\frac{1}{\sigma_{2} \sqrt{2 \pi}} e^{-\frac{\left(x-a_{2}\right)^{2}}{2 \cdot \sigma_{2}^{2}}}$ решается уравнение

$$
f_{1}(x)=f_{2}(x) \Rightarrow \frac{1}{\sigma_{1} \sqrt{2 \pi}} e^{-\frac{\left(x-a_{1}\right)^{2}}{2 \cdot \sigma_{1}^{2}}}=\frac{1}{\sigma_{2} \sqrt{2 \pi}} e^{-\frac{\left(x-a_{2}\right)^{2}}{2 \cdot \sigma_{2}^{2}}} .
$$

После преобразований получается квадратное уравнение

$$
\begin{aligned}
& \left(\sigma_{1}^{2}-\sigma_{2}^{2}\right) x^{2}+\left(2 \sigma_{2}^{2} a_{1}-2 \sigma_{1}^{2} a_{2}\right) x+ \\
& +\left(\sigma_{1}^{2} a_{2}^{2}-\sigma_{2}^{2} a_{1}^{2}+2 \sigma_{2}^{2} \sigma_{1}^{2} \ln \left(\sigma_{2} / \sigma_{1}\right)\right)=0
\end{aligned} .
$$

Решения $x_{1}, x_{2}\left(x_{1}<x_{2}\right)$ уравнения являются точками пересечения графиков.
Для параметров проводимого исследования математические ожидания неотицательны. Предварительно выявлены возможные формы фигуры пересечения графиков плотностей распределения для различных параметров листовой пластинки:

1) фигура состоит из одной части, когда техногенная кривая находится слева от фоновой (рис. 1):

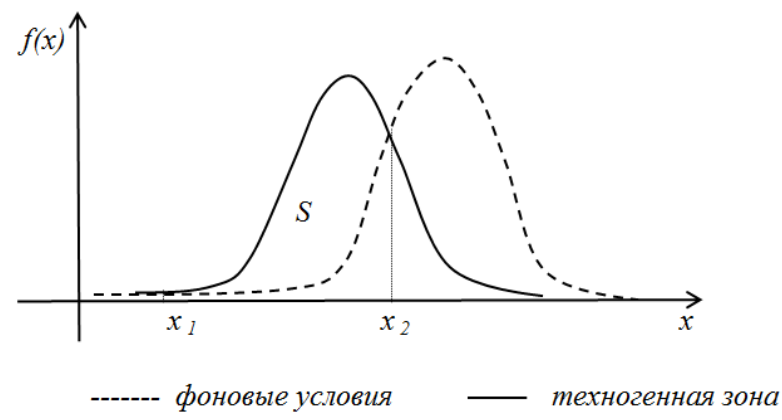

Рис. 1. Графики функций законов распределения исследуемого параметра листовых пластинок в случае нахождения техногенной кривой слева от фоновой

В этом случае площадь вычисляется по формуле $P_{i}=S=\left[\Phi\left(\frac{x_{2}-a_{2}}{\sigma_{2}}\right)-\Phi\left(\frac{x_{1}-a_{2}}{\sigma_{2}}\right)\right]-\left[\Phi\left(\frac{x_{2}-a_{1}}{\sigma_{1}}\right)-\Phi\left(\frac{x_{1}-a_{1}}{\sigma_{1}}\right)\right] ;$

2) фигура состоит из одной части, когда техногенная кривая находится справа от фоновой (рис. 2):

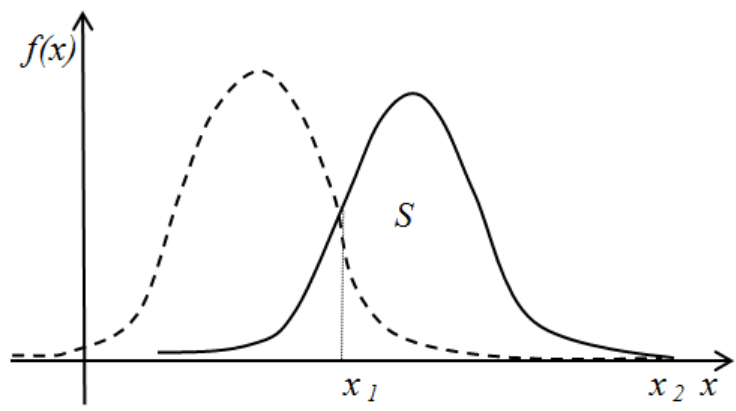

\section{фоновые условия - техногенная зона}

Рис. 2. Графики функций законов распределения исследуемого параметра листовых пластинок в случае нахождения техногенной кривой справа от фоновой

В этом случае площадь вычисляется по формуле $P_{i}=S=\left[\Phi\left(\frac{x_{2}-a_{2}}{\sigma_{2}}\right)-\Phi\left(\frac{x_{1}-a_{2}}{\sigma_{2}}\right)\right]-\left[\Phi\left(\frac{x_{2}-a_{1}}{\sigma_{1}}\right)-\Phi\left(\frac{x_{1}-a_{1}}{\sigma_{1}}\right)\right]$;

3) фигура состоит из двух частей, когда дисперсия техногенной зоны больше дисперсии фоновой (рис. 3):

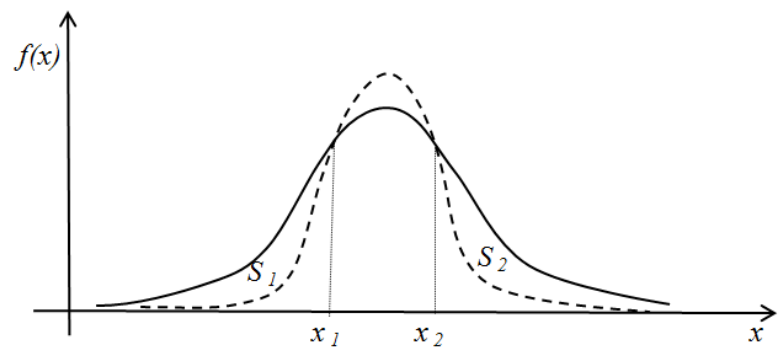

фоновые условия —- техногенная зона

Рис. 3. Графики функций законов распределения исследуемого параметра листовых пластинок в случае, когда дисперсия техногенной зоны больше дисперсии фоновой 
В этом случае площадь вычисляется по формулам

$\left(P_{i}\right)_{1}=S_{1}=\left[\Phi\left(\frac{x_{2}-a_{2}}{\sigma_{2}}\right)-\Phi\left(\frac{-\infty-a_{2}}{\sigma_{2}}\right)\right]-\left[\Phi\left(\frac{x_{2}-a_{1}}{\sigma_{1}}\right)-\Phi\left(\frac{-\infty-a_{1}}{\sigma_{1}}\right)\right]$ $\left(P_{i}\right)_{2}=S_{2}=\left[\Phi\left(\frac{+\infty-a_{2}}{\sigma_{2}}\right)-\Phi\left(\frac{x_{2}-a_{2}}{\sigma_{2}}\right)\right]-\left[\Phi\left(\frac{+\infty-a_{1}}{\sigma_{1}}\right)-\Phi\left(\frac{x_{2}-a_{1}}{\sigma_{1}}\right)\right]$.

$\mathrm{C}$ целью выявления, насколько отличаются значения $i$-го параметра адаптированных листовых пластинок относительно фоновых, предлагается использовать коэффициент глубины адаптивных изменений $\Gamma_{i}$.

Коэффициент глубины адаптивных изменений $\Gamma_{i}$ для $i$-го параметра находится по формуле $\Gamma_{i}=\frac{b-a}{b}=1-\frac{a}{b}$, где всегда $\mathrm{a}<\mathrm{b}$. Для сравнения значений параметра в фоновых условиях и техногенной зоне используются интервалы их наиболее вероятных значений (далее - интервалы), построенные по правилу трех сигм: $\left(\mathrm{a}_{1}-3 \sigma_{1} ; \mathrm{a}_{1}+3 \sigma_{1}\right)$, $\left(a_{2}-3 \sigma_{2} ; a_{2}+3 \sigma_{2}\right)$.

Возможны следующие варианты расположения интервалов (рис. 4):

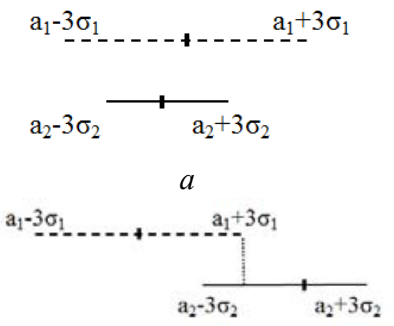

6

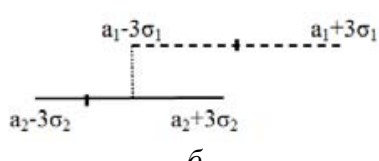

б

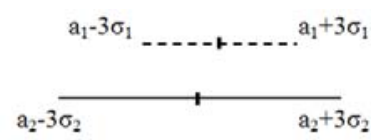

2
Рис. 4. Взаимное расположение интервалов наиболее вероятных значений параметра техногенной зоны относительно фоновых условий

1) интервал значений параметра техногенной зоны целиком содержится в интервале фоновых условий (рис. 4, a). В этом случае коэффициент глубины адаптивных изменений $\Gamma_{i}$ рассчитывается по формуле

$$
\Gamma_{i}=1-\frac{\min \left(a_{1}, a_{2}\right)}{\max \left(a_{1}, a_{2}\right)}
$$

2) интервал значений параметра техногенной зоны находится левее относительно интервала фоновых условий (рис. 4, б). В этом случае коэффициент глубины адаптивных изменений $\Gamma_{i}$ рассчитывается по формуле

$$
\Gamma_{i}=1-\frac{\frac{\left(a_{1}-3 \sigma_{1}\right)+\left(a_{2}-3 \sigma_{2}\right)}{2}}{a_{1}} ;
$$

3) интервал значений параметра техногенной зоны находится правее относительно интервала фоновых условий (рис. 4, в). В этом случае коэффициент глубины адаптивных изменений $\Gamma_{i}$ рассчитывается по формулам

$$
\Gamma_{i}=1-\frac{a_{1}}{\frac{\left(a_{1}+3 \sigma_{1}\right)+\left(a_{2}+3 \sigma_{2}\right)}{2}} ;
$$

4) интервал значений параметра фоновых условий целиком содержится в интервале техногенной зоны (рис. 4, 2). В этом случае коэффициент глубины адаптивных изменений $\Gamma_{i}$ рассчитывается по формуле:

$$
\begin{aligned}
& \left(\Gamma_{i}\right)_{1}=1-\frac{\frac{\left(a_{1}-3 \sigma_{1}\right)+\left(a_{2}-3 \sigma_{2}\right)}{2}}{a_{1}} ; \\
& \left(\Gamma_{i}\right)_{2}=1-\frac{a_{1}}{\frac{\left(a_{1}+3 \sigma_{1}\right)+\left(a_{2}+3 \sigma_{2}\right)}{2}} .
\end{aligned}
$$

Как уже отмечалось, итоговую величину коэффициента адаптивных изменений устанавливают по следующей формуле:

$$
A \Pi=\sum_{i=1}^{n} A \Pi_{i}=\sum_{i=1}^{n} P_{i} \times \Gamma_{i},
$$

где $n$ - количество показателей;

$P_{i}$ - вероятность адаптивных изменений $i$-го параметра;

$\Gamma_{i}-$ коэффициент глубины адаптивных изменений $i$-го параметра.

Заметим, что в случае когда для $i$-го параметра дисперсия техногенной зоны больше дисперсии фоновой (рис. 4, 2), коэффициент адаптивных изменений относительно этого параметра равен

$$
A \Pi_{i}=\sum_{k=1}^{2}\left(P_{i}\right)_{k} \times\left(\Gamma_{i}\right)_{k} .
$$

В качестве примера конкретного осуществления расчета приведем результаты определения коэффициента адаптивных изменений листовых пластинок березы повислой Betula pendula Roth., произрастающей в окружении ОАО «Гомельский химический завод» (Республика Беларусь, Гомель), с учетом морфометрических параметров и зольности (табл. 1-3).

\section{Таблица 1}

Длина, ширина, площадь и коэффициент флуктуирующей асимметрии (КФА) листовых пластинок березы повислой Betula pendula Roth.

\begin{tabular}{ccccccccc}
\hline \multirow{2}{*}{ № } & \multicolumn{7}{c}{ Морфометрические параметры листовых пластинок } \\
\cline { 2 - 8 } & \multicolumn{2}{c}{ ОАО «Гомельский химический завод» } & \multicolumn{3}{c}{ Национальный парк «Припятский» } \\
& Длина & Ширина & Площадь & КФА & Длина & Ширина & Площадь & КФА \\
\hline 1 & 58,21 & 38,54 & 1786,8 & 0,038 & 62,98 & 42,61 & 1855,09 & 0,094 \\
2 & 73,04 & 35,72 & 2158,65 & 0,060 & 65,62 & 49,48 & 2290,83 & 0,024 \\
3 & 65,65 & 39,95 & 1849,7 & 0,066 & 57,22 & 42,72 & 1746,96 & 0,058 \\
4 & 57,68 & 37,04 & 2010,48 & 0,043 & 64,82 & 47,17 & 2261,42 & 0,079 \\
5 & 69,07 & 38,1 & 1865,81 & 0,082 & 65,08 & 39,83 & 2033,32 & 0,032 \\
6 & 63,53 & 36,84 & 1699,39 & 0,102 & 70,92 & 49,37 & 2380,72 & 0,043 \\
7 & 72,37 & 31,54 & 2005,34 & 0,049 & 67,74 & 51,67 & 2481,84 & 0,060 \\
8 & 59,11 & 37,93 & 1688,94 & 0,098 & 71,00 & 46,69 & 2498,81 & 0,068 \\
9 & 59,32 & 32,41 & 1831,09 & 0,109 & 56,84 & 42,12 & 1807,85 & 0,008 \\
и т.д. & & & & & & & & \\
150 & 49,75 & 38,52 & 1371,03 & 0,085 & 59,48 & 40,3 & 1768,84 & 0,024 \\
\hline
\end{tabular}

Для проведения количественной оценки величины коэффициента адаптивных изменений на начальном этапе выявили наличие различий между исследуемыми параметрами в техногенных и фоновых условиях. В результате получено, что наблюдаемые t-значения болыше критических (1,97 для длины, ширины, площади; 2,002 - для сухой биомассы; 2,1 - 2,2 - для зольности при равных и различных дисперсиях), что указывает на наличие различий между сравниваемыми средними величинами. 
Таблица 2

Сухая биомасса листовых пластинок березы повислой Betula pendula Roth.

\begin{tabular}{ccc}
\hline \multirow{2}{*}{ № листа } & \multicolumn{2}{c}{ Сухая биомасса листовых пластинок, мг } \\
\cline { 2 - 3 } & $\begin{array}{c}\text { ОАО «Гомельский } \\
\text { химический завод» }\end{array}$ & $\begin{array}{c}\text { Национальный парк } \\
\text { «рипятский» }\end{array}$ \\
\hline 1 & 140,5 & 168,0 \\
2 & 142,9 & 157,1 \\
3 & 138,6 & 156,5 \\
4 & 139,4 & 170,2 \\
5 & 136,5 & 172,0 \\
6 & 142,5 & 169,2 \\
7 & 141,8 & 160,2 \\
8 & 142,7 & 165,3 \\
9 & 140,9 & 158,3 \\
и т.д. & &
\end{tabular}

Таблица 3

Зольность листовых пластинок березы повислой Betula pendula Roth.

\begin{tabular}{ccc}
\hline \multirow{2}{*}{ № листа } & \multicolumn{2}{c}{ Зольность листовых пластинок, \% } \\
\cline { 2 - 3 } & $\begin{array}{c}\text { ОАО «Гомельский } \\
\text { химический завод» }\end{array}$ \\
\hline 1 & 2,5 & 2,5 \\
2 & 2,0 & 2,6 \\
3 & 2,7 & 1,8 \\
4 & 2,8 & 1,5 \\
5 & 2,5 & 2,0 \\
6 & 2,7 & 2,0 \\
7 & 2,5 & 2,0 \\
8 & 2,0 & 1,8 \\
9 & 2,4 & \\
\hline
\end{tabular}

На предварительном этапе обработки данных по параметрам асимметрии листовых пластинок убедились во флуктуирующем характере асимметрии каждого признака и отсутствии или наличии направленной асимметрии и антисимметрии, а также достоверности различий между параметрами листа в техногенной и фоновой зонах и характере корреляционной связи между конкретными параметрами асимметрии листа отдельно с каждой стороны.

Как уже отмечалось, значение коэффициента адаптивных изменений, характеризующих адаптивный потенциал растений, определили как произведение вероятности и коэффициента глубины адаптивных изменений всех исследованных параметров листа.

Для длины и зольности листа березы повислой Betula pendula Roth., произрастающей в окружении ОАО «Гомельский химический завод» и на территории национального парка «Припятский», в табл. 4 представлены функции плотностей распределения вероятностей.

На рис. 5 изображены области перекрывания функций плотности распределения исследуемых параметров листа.

\section{Таблица 4}

Законы распределения для длины и зольности листовых пластинок березы повислой Betula pendula Roth.

\begin{tabular}{ccc}
\hline Место отбора проб & \multicolumn{2}{c}{ Законы распределения } \\
\cline { 2 - 3 } $\begin{array}{c}\text { ОАО «Гомельский } \\
\text { химический завод» }\end{array}$ & $f(x)=\frac{1}{7,09 \sqrt{2 \pi}} e^{-\frac{(x-59,40)^{2}}{2 \cdot 7,09^{2}}}$ & $f(x)=\frac{1}{0,29 \sqrt{2 \pi}} e^{-\frac{(x-2,5)^{2}}{2 \cdot 0,29^{2}}}$ \\
$\begin{array}{c}\text { Национальный парк } \\
\text { «Припятский» }\end{array}$ & $f(x)=\frac{1}{4,71 \sqrt{2 \pi}} e^{-\frac{(x-61,55)^{2}}{2 \cdot 4,71^{2}}}$ & $f(x)=\frac{1}{0,37 \sqrt{2 \pi}} e^{-\frac{(x-2,0)^{2}}{2 \cdot 0,37^{2}}}$ \\
\hline
\end{tabular}

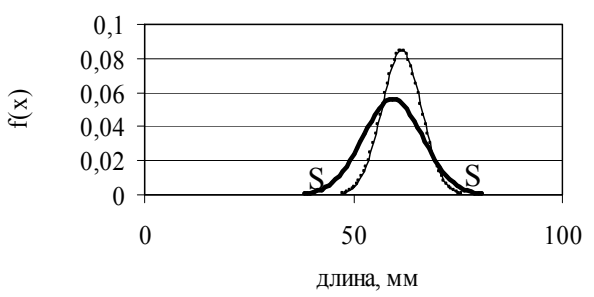

— ОАО «Гомельский химический завод»

- - Национальный парк "Припятский"

$a$

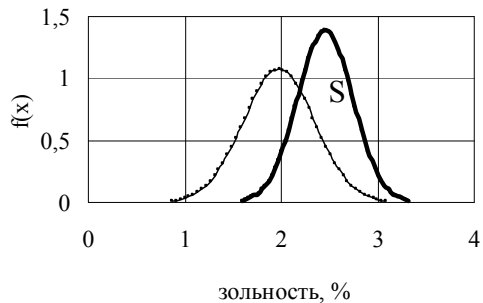

—ОАО «Гомельский химический завод»

- - Национальный парк "Припятский" $\sigma$

Рис. 5. Графики функций законов распределения длины (а) и зольности (б) листовых пластинок березы повислой Betula pendula Roth., произрастающей в техногенных и фоновых условиях 
Если распределение исследуемого параметра не является нормальным, используем логнормальное распределение (для коэффициента флуктуирующей асимметрии).

В случае коэффициента флуктуирующей асимметрии (обозначим ee $X$ ) получили, что гистограмма распределения вероятностей (рис. 6) и проверка на значимость асимметрии и эксцесса нормального

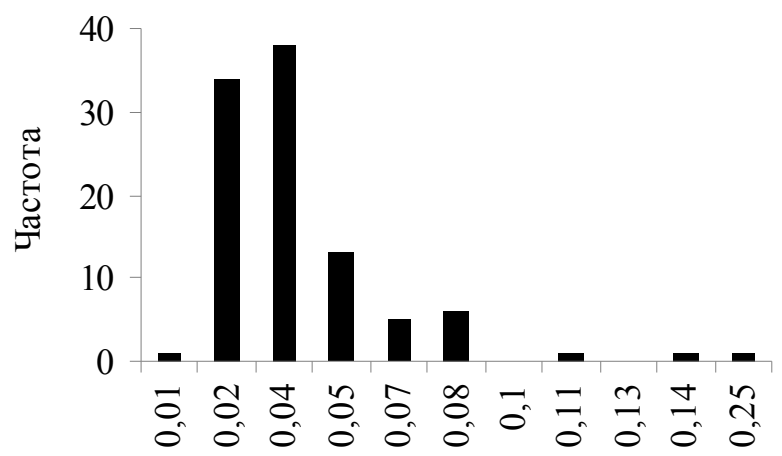

Рис. 6. Частоты распределения коэффициента флуктуирующей асимметрии параметров листовых пластинок березы повислой Betula pendula Roth. на территории национального парка «Припятский»

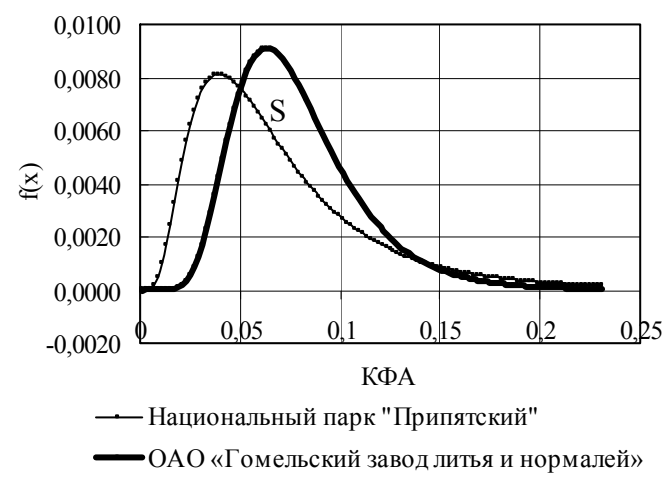

$a$ - логнормальное распределение

Рис. 7. Кривые распределения случайной величины коэффициента флуктуирующей асимметрии параметров листовых пластинок березы повислой Betula pendula Roth. распределения позволяют отвергнуть предположение о нормальном распределении случайной величины $X$ и выдвинуть гипотезу о логнормальном распределении исследуемой случайной величины. Это следует из того, что значения асимметрии и эксцесса, вычисленные по выборке, в большинстве случаев больше критического значения соответствующей статистики, и асимметрия и эксцесс для каждой из техногенных зон значительно отличаются от нуля.

Величина вероятности адаптивных изменений по коэффициенту флуктуирующей асимметрии равна площади $S$ фигуры, заключенной между графиками плотностей распределения техногенной и фоновой вероятностей. Поскольку преобразование натурального логарифма строго монотонно, то равенство значений функции распределения случайной величины $X$ и еe натурального логарифма правомерно использовать при вычислении указанной площади.

Так, например, графики плотности логнормального распределения вероятности коэффициента флуктуирующей асимметрии для национального парка «Припятский» и ОАО «Гомельский завод литья и нормалей» приведены на рис. 7, $a$.

Для определения коэффициента глубины адаптивных изменений по коэффициенту флуктуирующей асимметрии $\left(\Gamma_{\phi a}\right)$ необходимо сравнить интервалы наиболее вероятных значений показателя в техногенной зоне и в фоновых условиях.

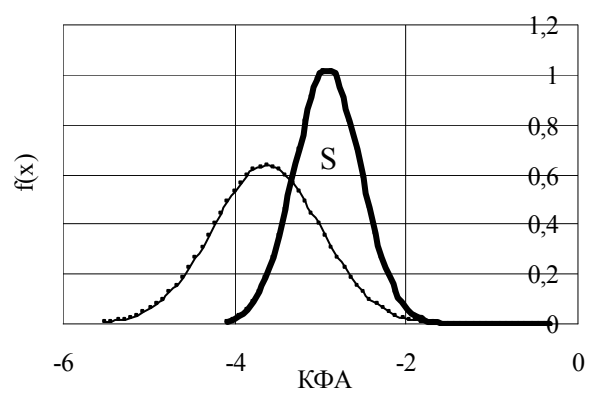

- $\mathrm{OAO}$ «Гомельский завод литья и нормалей» _. Национальный парк "Припятский" $\sigma$ - нормальное распределение
Поскольку распределение показателя асимметрично, то в такой ситуации полагаться на среднее и стандартное отклонение нельзя. Для описания таких данных воспользуемся 5-й и 95-й процентилями в качестве границ интервалов. Глубину (Гфа) рассчитали как разность единицы и отношения среднего интервала изменений показателя в фоновых условиях к среднему тех значений показателя в техногенных зонах, которые не попали в интервал фоновых значений.

Итоговые значения вероятности и коэффициента глубины адаптивных изменений представлены в табл. 5.

\section{Таблица 5}

Значения вероятности и коэффициента глубины адаптивных изменений параметров листовых пластинок березы повислой Betula pendula Roth.

\begin{tabular}{|c|c|c|c|c|c|c|}
\hline \multirow[b]{2}{*}{ Место отбора проб } & \multicolumn{6}{|c|}{ Параметры листовых пластинок } \\
\hline & Длина & Ширина & Площадь & $\begin{array}{c}\text { Сухая } \\
\text { биомасса }\end{array}$ & Зольность & $\begin{array}{c}\text { Коэффициент } \\
\text { флуктуирующей } \\
\text { асимметрии }\end{array}$ \\
\hline \multicolumn{7}{|c|}{ Вероятность адаптивных изменений } \\
\hline $\begin{array}{c}\mathrm{OAO} \text { «Гомельский } \\
\text { химический } \\
\text { завод» }\end{array}$ & $\frac{0,195}{0,029}$ & 0,839 & 0,248 & 0,999 & 0,539 & 0,521 \\
\hline \multicolumn{7}{|c|}{ Коэффициент глубины адаптивных изменений } \\
\hline $\begin{array}{c}\mathrm{OAO} \text { «Гомельский } \\
\text { химический } \\
\text { завод» }\end{array}$ & $\frac{0,304}{0,213}$ & 0,325 & 0,512 & 0,164 & 0,382 & 0,527 \\
\hline \multicolumn{7}{|c|}{ Коэффициент адаптивных изменений } \\
\hline $\begin{array}{c}\mathrm{OAO} \mathrm{«Гомельский} \\
\text { химический } \\
\text { завод» }\end{array}$ & 0,065 & 0,273 & 0,127 & 0,164 & 0,206 & 0,275 \\
\hline
\end{tabular}


Итоговое значение величины коэффициента адаптивных изменений листовых пластинок березы повислой Betula pendula Roth., произрастающей на территории ОАО «Гомельский химический завод», равно 1,11 .

При интерпретации полученной величины учитываем, что ее значение изменяется в общем случае от 0 до 6 (т.к. учитывались 6 параметров листа).

Также разработана оценочная шкала оценки величины коэффициента адаптивных изменений:

- $A \Pi \in(0 ; 1,2)$ - реализация адаптивных изменений исследуемых представителей незначительна;

- при $A \Pi \in(1,3 ; 2,5)$ - растения демонстрируют умеренную способность приспосабливаться к техногенным условиям;

- если $A \Pi \in(2,6 ; 3,8)$ - адаптивные изменения реализованы значительно;

- сильные адаптационные изменения - $A \Pi \in(3,9 ; 5,0)$ и максимально возможные $-A \Pi \in(5,0 ; 6,0)$.

В данном конкретном случае адаптивные изменения параметров листовых пластинок березы повислой Betula pendula Roth., произрастающей на территории ОАО «Гомельский химический завод», характеризуются как незначительные.

\section{Выводы}

Предложенный способ позволяет количественно определить величину коэффициента адаптивных изменений параметров растений под влиянием факторов внешней среды, в т.ч. техногенных, относительно фоновых условий. Расчет осуществляется путем определения вероятности и коэффициента глубины адаптивных изменений, при этом величина вероятности адаптивных изменений равна площади фигуры, заключенной между графиками плотностей распределения техногенной и фоновой вероятностей, а для определения коэффициента глубины адаптивных изменений необходимо сравнить интервалы наиболее вероятных значений показателя в техногенной зоне и в фоновых условиях. Итоговый коэффициент адаптивных изменений определяется как сумма коэффициентов адаптивных изменений относительно всех исследуемых параметров, количество которых связано с целями, задачами, техническими возможностями конкретного исследования.

\section{References}

Aseeva, T. A., Karacheva, G. S. (2015). Priemi povichenia adaptivnogo potensiala zernovich kultur $\mathrm{v}$ usloviach Srednego Priamurya [Methods of increasing the grain crops adaptive potential in the Middle Amur region], http://www.old.dalgau.ru (in Russian).

Bezel, V. S., Pozolotina, V. S., Belsky, V. N., Zhuykova, T. V. (2001). Izmenchivost populyatsionnyih parametrov: adaptatsiya $\mathrm{k}$ toksicheskim faktoram sredyi [Variation of population parameters: adaptation to the toxic environmental factors]. Ekologiya, 6, 447-453 (in Russian).

Mazurkin, P. M., Kudryasheva, A. I. (2015). Sposob analiza dinamiki rosta $\mathrm{V}$ ontogeneze zagraznenich listev berezi okolo avtomobilnoi dorogi: patent RF 2597643, MPK G 01 D 21/00 [Method of analysis the growth dynamics polluted birch leaves in ontogenesis around the road], http://www.findpatent.ru (in Russian).

Zhuchenko, A. A. (2008). Adaptivnoe rastenievodstvo (ecologo-geneticheskie osnovi). Teoria i praktica [Adaptive plant growing (ecological and genetic basis). Theory and practice]. Moscow (in Russian). 